\title{
Digital scanning and BIM modeling for modern architecture preservation: the Oscar Niemeyer's Church of Saint Francis of Assisi
}

\section{SIGRADI2018 TECHNOPOLITICAS \\ xxii congresso da sociedade iberoamericana de gráfica digital 22th conference of the iberoamerican society of digital graphics 07|08|09|novembro|2018 iau usp | são carlos | sp br}

\author{
Camila Kimi Cogima \\ UNICAMP, School of Technology | Brazil | camila.cogima@pos.ft.unicamp.br \\ Pedro V. V. de Paiva \\ UNICAMP, School of Technology | Brazil | pedro.paiva@pos.ft.unicamp.br \\ Eloisa Dezen-Kempter \\ UNICAMP, School of Technology | Brazil | elo@ft.unicamp.br
}

Marco Antonio G. De Carvalho

UNICAMP, School of Technology | Brazil | magic@ft.unicamp.br

\begin{abstract}
The Building Information Modelling (BIM) technology enabled improvement in the design, construction and maintenance stages highly. In the field of existing buildings, including historical assets, this technology has not yet had the same impact. This paper presents a methodology to create an intelligent digital model for an outstanding building from modern architecture in Brazil using multiple reality-based technologies. The fusion of the different point cloud raw data generated a high-resolution Dense Surface Model (DSM), the base of an accurate and detailed parametric Model. This study demonstrated the potential of digital surveying, including low-cost sensors, and BIM for built heritage documentation.
\end{abstract}

Keywords: Reality-based surveying; Point cloud; As-is model; Building Information Modelling; Modern Heritage.

\section{INTRODUÇÃO}

O desenvolvimento de novas tecnologias de informação e comunicação para o setor de Construção Civil, dentre elas a Modelagem da Informação da Construção (BIM), tornou-se um fator decisivo de avanço neste setor. Essa tecnologia possibilitou a ampliação de processos colaborativos em todas as fases do projeto, inclusive nas etapas de operação, uso e manutenção, gestão de instalações, assim como, em análises e simulações visando a sustentabilidade da construção.

A premissa de que BIM é uma tecnologia fundamental no processo de tomada de decisão e no fluxo de informações, entre os colaboradores nas fases de projeto e construção, mostrou ser funcional para novos edifícios, mas ainda deixa várias lacunas de aplicabilidade para edifícios existentes (Quattrini et al., 2017). Isso se deve principalmente pela dificuldade de modelagem paramétrica para a representação de elementos arquitetônicos complexos e irregulares, típicos de edificações históricas.

Vários trabalhos têm explorado o levantamento digital para a representação de edificações históricas (Sun, Cao, 2015; Hou, 2016; Castagnetti et al., 2017; entre outros). Dore e Murphy (2012), em um trabalho seminal, propuseram a integração de dados de escaneamento a laser e imagens, desenvolvendo uma metodologia denominada Modelagem da Informação da Construção Histórica (HBIM). Sua abordagem fundamentou-se em um método semiautomático de coleta e modelagem de elementos arquitetônicos representativos das edificações históricas de seu país, criando uma biblioteca pré-definida de objetos paramétricos, utilizando linguagem de programação.

Quanto à criação de bibliotecas de componentes históricos, Antón et al. (2018) se opõem alegando que cada edifício histórico tem suas peculiaridades. Seus elementos são substancialmente diferentes, os materiais empregados são específicos e a arquitetura é singular, o que torna-se afanoso a utilização de bibliotecas BIM padronizadas para retratar com precisão o objeto histórico.

Quanto à abordagem metodológica para a criação de modelos inteligentes para edificações existentes, Volk et al. (2014) e Tang et al. (2010) descrevem as três etapas fundamentais para o processo: (1) levantamento de dados (espaciais e documentais); (2) processamento de dados e criação do modelo denso de superfície (DSM); e (3) modelagem BIM (a partir do reconhecimento de componentes no DSM e sua etiquetagem semântica). Portanto, o DSM é a ponte entre o objeto real e sua representação BIM.

Este artigo apresenta uma abordagem baseada em Volk et al. (2014) e Tang et al. (2010), para a criação do Modelo BIM para um edifício representativo da moderna arquitetura brasileira, a Igreja de São Francisco de Assis no conjunto arquitetônico da Pampulha em Belo Horizonte. Nossa abordagem emprega dois sensores na varredura digital: um escâner a laser terrestre (TLS) e um veículo aéreo não tripulado (VANT) de baixo custo, cuja acurácia do levantamento foi comparada com a varredura 
a laser. A forma peculiar desta edificação suscitou um enfoque de modelagem BIM baseado em massas.

\section{SOBRE A CAPTURA DIGITAL}

Concebidos inicialmente para serviços militares com 0 objetivo de mapear o território a ser explorado, os VANTs tornaram-se também equipamentos úteis para para capturas a curta distância (Murtiyoso, Grussenmeyer, \& Freville, 2017).

Uma década atrás, Everaerts (2008) já relatava sobre programas, regulamentações e classificação de VANTs como instrumento de sensoriamento remoto, especialmente na arqueologia.

Nex \& Remondino (2014) classificam os VANTs quanto as suas capacidades aerodinâmicas e físicas em três tipos: (1) mais leve que o ar; (2) asa rotativa, elétrica ou com motor a combustão; e (3) asa fixa, sem motor, elétrica ou com ICE (Internal Combustion Engine). Os drones se encaixam no segundo tipo.

Para levantamentos aplicados à documentação do patrimônio cultural, Themistocleous et al. (2015) afirmam que os VANTs podem ser um recurso eficiente, não invasivo e de baixo custo, além de serem acessíveis, confiáveis e de fácil utilização.

Os dados capturados são processados em programas de reconstrução densa de superfície (Dense Stereo Matching), que aplicam a maioria dos princípios da fotogrametria e são capazes de produzir dados precisos de um objeto a partir de um conjunto de imagens não calibradas.

O processamento dos dados coletados em levantamentos com imagens em dados tridimensionais, a partir de uma sequência de vídeo ou a partir de um conjunto de dados fotográficos não estruturados, foi exaustivamente abordado em pesquisas no campo da Ciência da Computação nos últimos anos, resultando na criação de ferramentas com cada vez mais recursos. Estas ferramentas estão sendo amplamente utilizadas para a documentação patrimonial, devido à baixa exigência de capacidade de hardware, ampla gama de aplicações e facilidade de uso (Dellepiane et al., 2013).

A precisão dos DSMs, gerados a partir de dados fotogramétricos de VANTs, são influenciados por diversos fatores. Clapuyt, Vanacker \& Van Oost (2016) apontam que a distância focal é um fator decisivo. Rock, Ries \& Udelhoven (2011) mostram como a altitude do voo pode interferir na qualidade final, e Nouwakpo, Weltz \& McGwire (2016) afirmam que a qualidade da câmera e a qualidade da imagem são cruciais para um modelo preciso.

\section{A FORMA PECULIAR DA IGREJA SÃO FRANCISCO DE ASSIS NA PAMPULHA}

O Conjunto Moderno da Pampulha é formado por uma paisagem que agrega quatro edifícios articulados em torno do lago urbano artificial de mesmo nome (Fig. 1), listada em 2016 como patrimônio da Humanidade pela UNESCO, por seu excepcional valor universal.

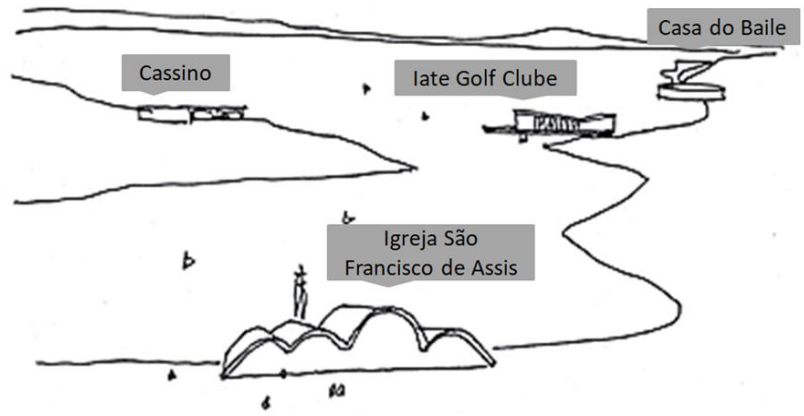

Figura 1: Croqui de Oscar Niemeyer para o Conjunto da Pampulha.

O arquiteto Oscar Niemeyer projetou os quatro prédios do conjunto (Casa de Baile, Igreja de São Francisco de Assis, Cassino e late Golf Clube) na década 1940. Os experimentos em concreto armado nas obras de Niemeyer na Pampulha, exploraram as possibilidades expressivas da curva, superando a racionalidade construtiva do movimento moderno. Na Igreja de São Francisco de Assis, Niemeyer deixou a laje sobre pilotis e criou uma série de abóbadas parabólicas de concreto, até então usada apenas em hangares. Estes vãos funcionam ao mesmo tempo que cobertura e fechamento (Fig. 2).

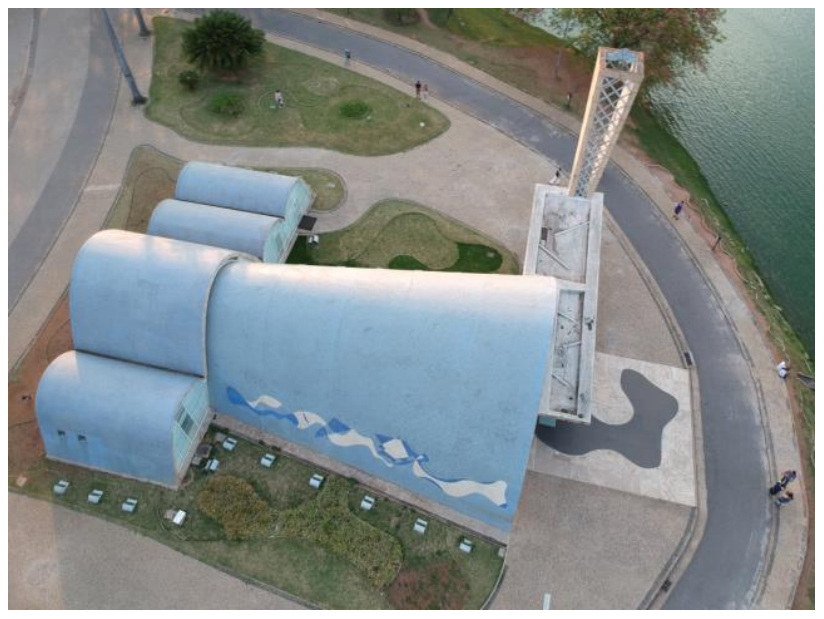

Figura 2: Foto Aérea da Fachada Lateral da Igreja São Francisco de Assis. Fonte: Autores.

\section{ABORDAGEM METODOLÓGICA}

A estrutura metodológica empregada para a reconstrução geométrica 3D BIM da Igreja de São Francisco de Assis da Pampulha é composta de 5 passos ilustrados na figura 3. 


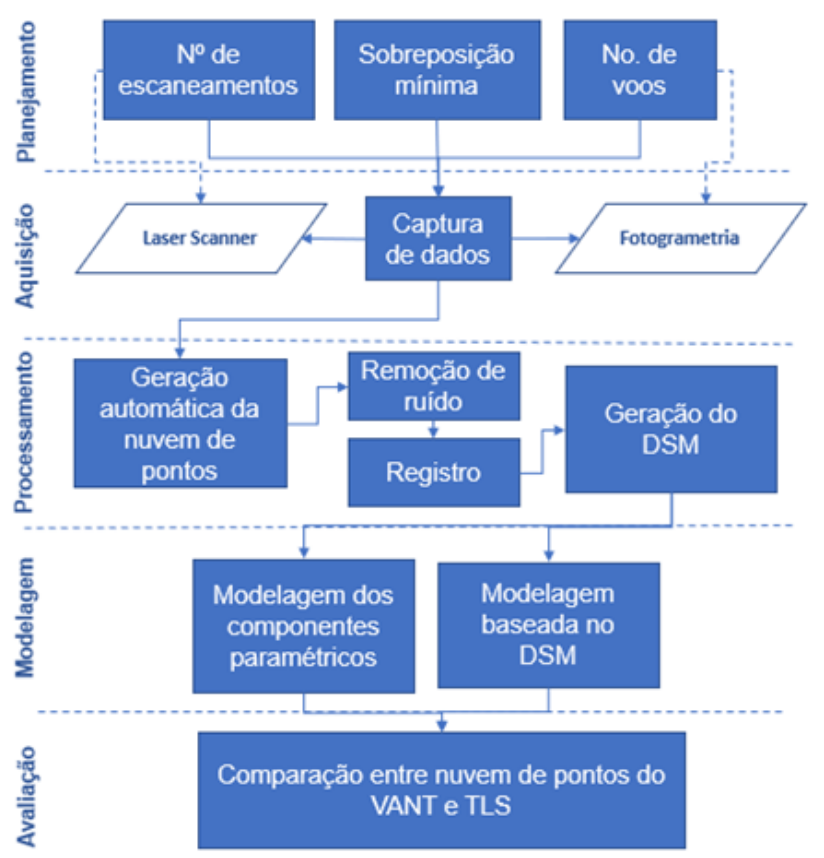

Figura 3: Fluxograma das etapas para a geração do modelo BIM. Fonte: Autores.

A utilização de equipamentos para a captura à curta distância de dados de edificações, especialmente com arquitetura complexa, ainda não dispõe de metodologia definida (Nex \& Remondino, 2014). Apesar das especificidades de cada objeto, alguns parâmetros são fundamentais no planejamento da aquisição com VANTs: (a) número de voos; (b) percurso de voo; (c) ângulo de captura; (d) sobreposição mínima desejada e (e) segurança do equipamento.

Com base do planejamento de aquisição, as tomadas fotográficas são realizadas e o DSM é gerado. Em seguida, é necessário realizar a remoção do ruído e reduzir o tamanho do arquivo para viabilizar seu uso.

Os dados tridimensionais provenientes do DSM não possuem orientação quanto a localização geoespacial e identificação dos elementos capturados. Isso torna a Modelagem da Informação da Construção etapa importante para fornecer inteligência a esses dados.

Para garantir a precisão do modelo final é necessário a etapa de avaliação. Nessa pesquisa utilizamos a nuvem de pontos gerada pelo escâner a laser terrestre (TLS) como parâmetro para avaliar a acurácia do levantamento com o VANT.

Empregou-se a varredura digital por escaneamento com TLS e fotogrametria aérea usando um modelo de VANT amador. Os equipamentos estão especificados no quadro 1.
Quadro 1: Especificação dos equipamentos empregados

\begin{tabular}{ll}
\hline Tecnologia & Equipamento \\
\hline Escaneamento & Laser Escâner Terrestre, modelo Focus3D \\
a laser & X130, Faro Technologies, equipado com \\
& câmera a cores de 70MP, alcance até \\
& 130m, campo de visão 300ํvertical e 360ำ \\
& horizontal. \\
\hline Fotogrametria & Quadricóptero modelo Spark, DJI, com \\
Digital aérea & câmera acoplada de 12MP (3968×2976), \\
& lente F/2.6, 25 mm (equivalente ao \\
& formato 35 mm), FOV 81.9 \\
\hline
\end{tabular}

Para a modelagem BIM foi utilizada a ferramenta autoral REVIT da Autodesk. Como ferramentas de apoio foram empregadas o Autocad (manipulação da nuvem de pontos) e Recap Pro (conversão de arquivos).

O software CloudCompare foi responsável pela união das nuvens de pontos dos diferentes sensores, sua decimação e limpeza de ruídos, assim como a checagem de acurácia.

\section{RESULTADOS E DISCUSSÃO PLANEJAMENTO DA CAPTURA}

As posições do escaneamento a laser levaram em consideração a sobreposição necessária (>50\%) para registro das nuvens de pontos. Foram consideradas também as áreas de oclusão e forma peculiar da cobertura. As posições do TLS podem ser observadas na Figura 4.

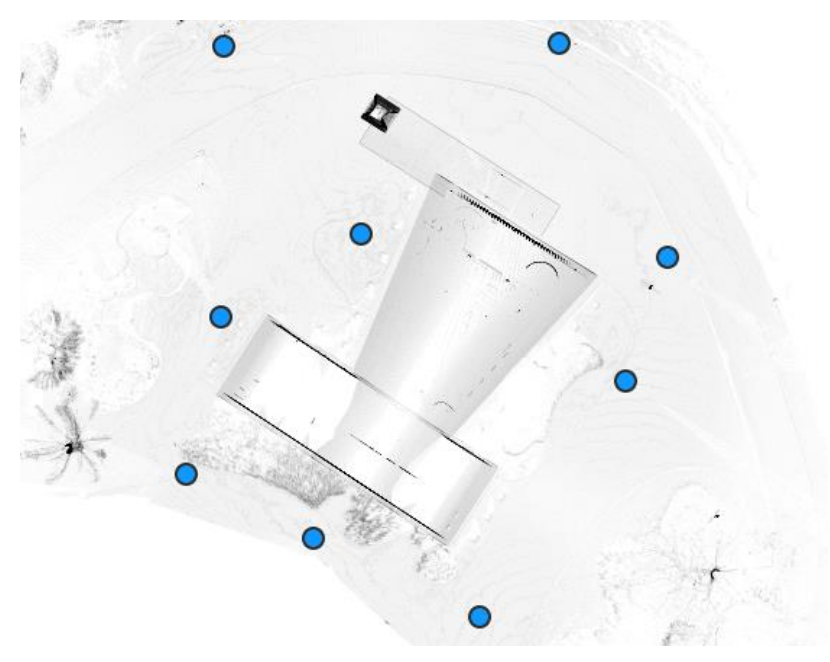

Figura 4: Posições do TLS para a varredura da igreja. Fonte: Autores.

A localização da Igreja São Francisco de Assis, junto à lagoa da Pampulha e a proximidade com o Aeroporto Carlos Drummond de Andrade, restringiu as possibilidades de captura aérea. A interferência de sinal de GPS entre o controle e o VANT impediu o percurso do voo sobre a lagoa. Assim, optou-se por trajetos em linhas paralelas nos sentidos longitudinal e transversal, com o eixo inclinado a $90^{\circ}$, conforme a figura 4. 

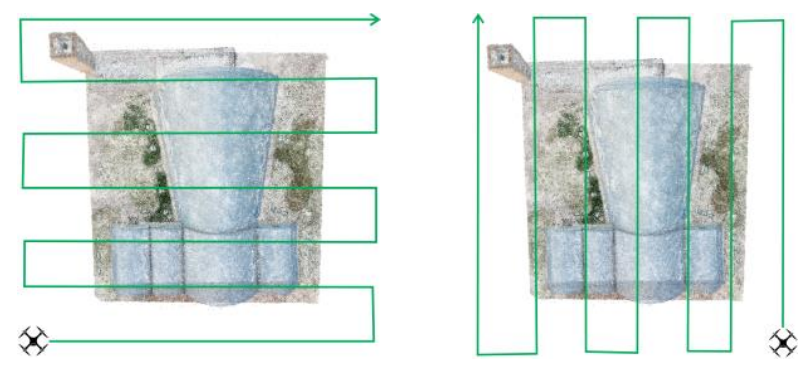

Figura 4: Planejamento da captura - sentido transversal (dir.) e sentido longitudinal (esq.). Fonte: Autores.

\section{CAPTURA DOS DADOS}

O quadricóptero amador Spark da empresa DJI foi empregado para a captura fotográfica. Ele foi operado manualmente, voou entre 20-30 metros do solo, e os disparos foram automatizados para cada 5 s, gerando 300 fotos (figura 6).

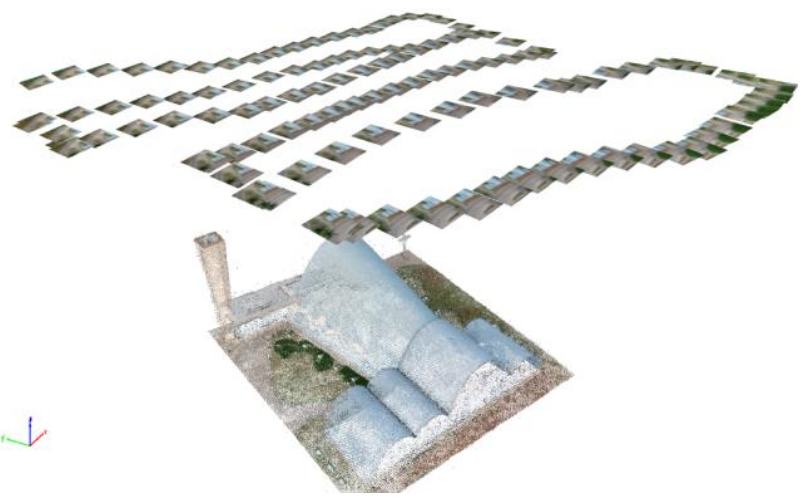

Figura 6: Trajeto e aquisição das fotografias com o VANT no sentido longitudinal. Fonte: Autores.

O TLS empregado para a varredura digital foi o modelo Focus3D X130 da Faro Technologies. A resolução foi configurada para $1 / 5$ e a qualidade $4 x$ (refere-se ao tempo de escaneamento destinado a capturar um ponto, neste caso 8 us por ponto escaneado), para distância entre pontos de $7,7 \mathrm{~mm}$ a cada $10 \mathrm{~m}$.

\section{PROCESSAMENTO}

A fase de processamento foi executada através de dois programas: Pix4Dmapper da Pix4D e Scene, software proprietário da Faro.

As fotos do VANT foram processadas pelo Pix4Dmapper, com cerca de 21 minutos para o processamento inicial, 23 minutos para a densificação da nuvem de pontos e 4 minutos e meio para a geração da malha texturizada 3D. O resultado foi a geração de uma nuvem de pontos com densidade absoluta de 602.013 pontos, e 327,17 pontos por $\mathrm{m}^{3}$, exportada em formato .OBJ

As varreduras digitais individuais do TSL foram importadas e registradas no software Scene e exportadas em formato .PTS

Os arquivos gerados pelos dois sistemas foram importados no software open source CloudCompare para serem alinhados e mesclados em uma única nuvem de pontos, formando um modelo híbrido.

\section{MODELAGEM}

O modelo BIM foi criado usando o DSM como base. Ele foi exportado do CloudCompare em formato .LAS e importado para o ReCAP (Autodesk) visando gerar o arquivo formato .RCP para a importação em outras plataformas Autodesk, como o Autocad e o REVIT.

No Autocad a nuvem foi recortada para viabilizar a modelagem dos componentes individualizados assim como para a extração de arestas para a modelagem das paredes. Além disso, no ambiente de criação de famílias (.RFA) o REVIT não aceita 0 arquivo de nuvem de pontos, sendo necessário empregar o arquivo gerado no Autocad. Os arquivos .RCP e .DWG foram inseridos no REVIT como um link. A Fig. 7 apresenta o corte perspectivado do modelo gerado no REVIT com a sobreposição da nuvem de pontos da captura do ambiente interno da Igreja.

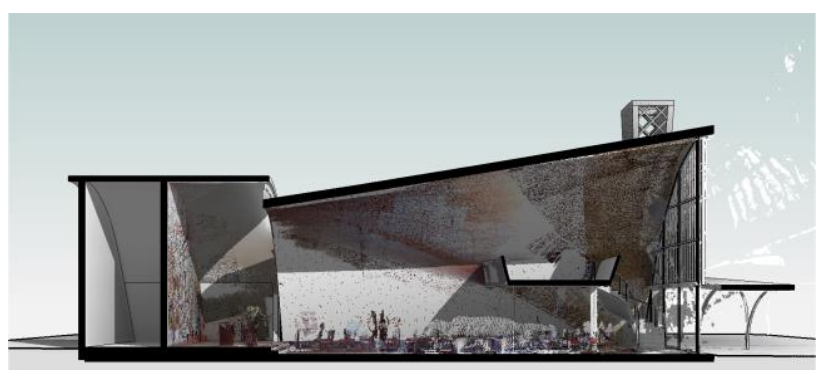

Figura 7: Corte Perspectivado do Modelo BIM da Igreja de São Francisco de Assis. Fonte: Autores.

Cada componente foi etiquetado usando a estrutura primitiva do REVIT.

O grande desafio da modelagem foram as estruturas parabólicas de cobertura. Como elas trabalham ao mesmo tempo como paredes e cobertura, esses elementos foram etiquetados como cobertura, possibilitando sua conexão com as paredes planas internas e das fachadas. Nos vãos parabólicos empregou-se a ferramenta de modelagem de massa do REVIT em função da espessura variável e da curvatura.

O modelo final, como os materiais aplicados, está apresentado nas Figuras 8 e 9.

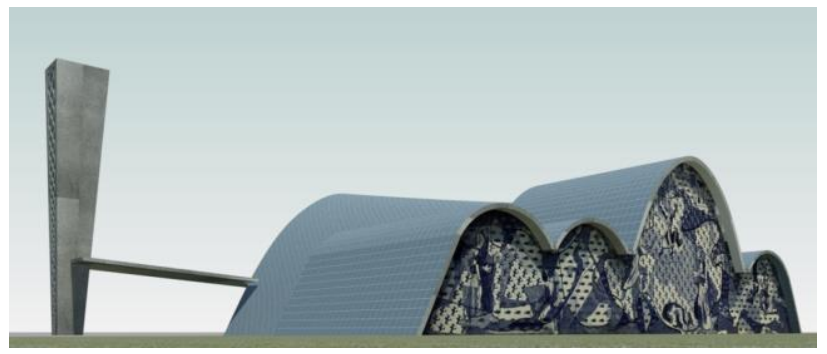

Figura 8: Fachada Posterior. Fonte: Autores. 


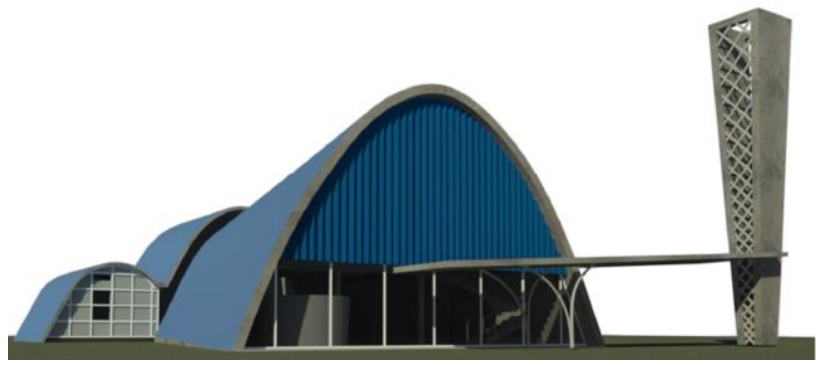

Figura 9: Fachada Principal. Fonte: Autores.

É possível visualizar todos componentes modelados em destaque na perspectiva explodida da Figura 10.

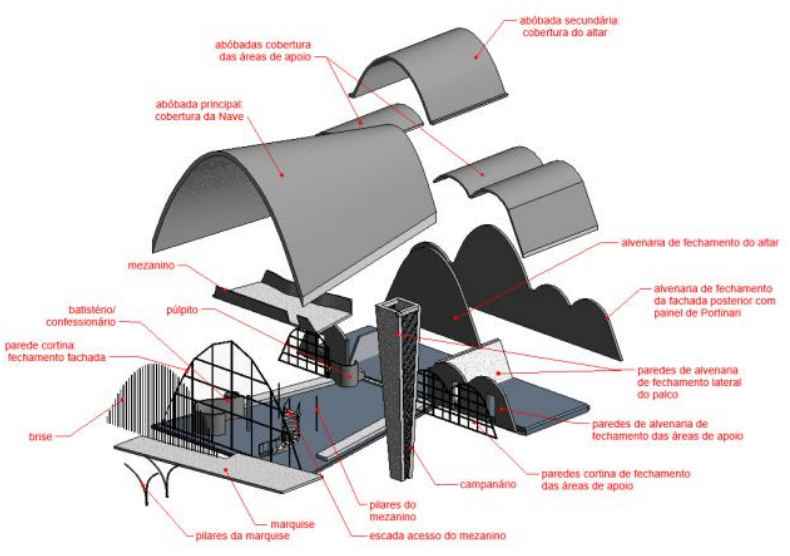

Figura 10: Componentes Arquitetônicos da Igreja de São Francisco de Assis. Fonte: Autores.

\section{AVALIAÇÃO}

A nuvem híbrida possibilita a complementação de informações coletadas pelos diferentes sensores, preenchendo áreas de oclusão de cada sistema.

A sobreposição das nuvens em um único sistema de coordenadas permite ainda realizar testes de precisão relacionando os pontos das diferentes nuvens e medindo suas distâncias. Para a avaliação foi adotada a nuvem do TSL como referência de precisão.

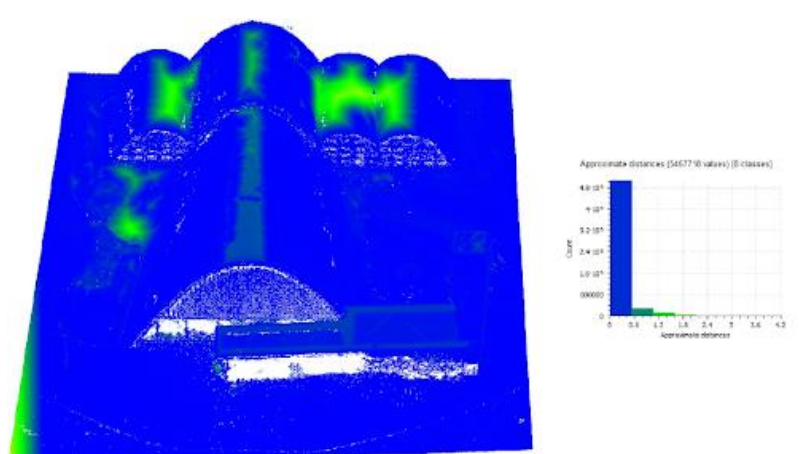

Figura 11: Comparação entre nuvens de pontos VANT e TLS. Fonte: Autores.

A figura 11 ilustra a nuvem híbrida da Igreja São Francisco, e o histograma de comparação. Percebe-se em mais de $90 \%$ dos pontos um alto índice de sobreposição, com distâncias aproximadas menores que $0,6 \mathrm{~mm}$ (cor azul). Os pontos onde as distâncias foram maiores (verde claro) são os locais que não foram cobertos pelo TLS.

\section{CONCLUSÃO}

Este trabalho propôs uma estrutura metodológica detalhada para a criação do modelo BIM para um exemplar singular da arquitetura moderna brasileira.

Para o levantamento do edifico foram empregados dois tipos sensores, um escâner a laser terrestre, com grande precisão na coleta de dados, e uma câmera não profissional acoplada a um VANT amador.

Quanto à aquisição, foram propostos voos cruzados sobre a Igreja, com a câmera em ângulo normal ao objeto para cobrir áreas de difícil visualização e que não conseguiram ser varridas pelo TLS, como o topo das coberturas e as áreas de intersecção das abóbadas (áreas verdes na Figura 11). Esse tipo de voo possibilita uma grande área de sobreposição de imagens, e, portanto, a geração de uma nuvem mais densa e precisa.

A avaliação das nuvens, através de sobreposição e cálculo de distâncias entre pontos, mostrou que o VANT, um equipamento de baixo custo, teve um desempenho excelente. A nuvem gerada por esse equipamento teve mais de $90 \%$ de sobreposição com o TLS, e onde os resultados foram baixos, é exatamente onde não havia cobertura pelo equipamento de alta precisão. Isso comprova que a captura com VANTs amadores, considerados menos precisos em comparação com TLSs (Xu et al., 2016; Faltýnová et al., 2016; Moussa, AbdelWahab \& Fritsch, 2012), geram dados com alto grau de confiança, mesmo sistemas amadores com câmeras não profissionais, graças ao poder de restituição fotogramétrica dos modernos software. Além disso, seu custo é, em média, 100 vezes menor em relação ao TLS, fator crucial na tomada de decisão.

O modelo BIM gerado a partir do DSM constitui uma forma de documentação digital para o patrimônio arquitetônico, no qual torna-se possível reunir todos os dados coletados em uma base estruturada, orientada aos componentes da edificação, abrangendo suas propriedades formais, materiais, históricas, e podendo contemplar seu ciclo de vida.

Pontos a serem explorados em trabalhos futuros é como estruturar no modelo BIM a informação patrimonial.

\section{AGRADECIMENTOS}

Agradecemos à Fundação de Amparo à Pesquisa do Estado de São Paulo (FAPESP) pelo auxílio financeiro ao projeto de pesquisa processo $n^{\circ}$ 2016/04991-0, e pelas bolsas de mestrado, processos no 2017/02787-9 e 2017/01237-5

\section{REFERÊNCIAS}

Antón, D., Medjdoub, B., Shrahily, R., \& Moyano, J. (2018). Accuracy evaluation of the semi-automatic 3D modeling for historical building information models. International Journal of Architectural Heritage, 12(5), 790-805..

Castagnetti, C., Dubbini, M., Ricci, P. C., Rivola, R., Giannini, M., \& Capra, A. (2017). Critical issues and key points from the survey to the creation of the Historical Building Information Model: the case of Santo Stefano Basilica. The International Archives of Photogrammetry, Remote Sensing and Spatial Information Sciences, 42, 467. 
Clapuyt, F., Vanacker, V., \& Van Oost, K. (2016). Reproducibility of UAV-based earth topography reconstructions based on Structure-from-Motion algorithms. Geomorphology, 260, 415.

Dellepiane, M., Dell'Unto, N., Callieri, M., Lindgren, S., \& Scopigno, R. (2013). Archeological excavation monitoring using dense stereo matching techniques. Journal of Cultural Heritage, 14(3), 201-210.

Dore, C., \& Murphy, M. (2012). Integration of Historic Building Information Modeling (HBIM) and 3D GIS for recording and managing cultural heritage sites. In Virtual Systems and Multimedia (VSMM), 2012 18th International Conference on (pp. 369-376). IEEE.

Everaerts, J. (2008). The use of unmanned aerial vehicles (UAVs) for remote sensing and mapping. The International Archives of the Photogrammetry, Remote Sensing and Spatial Information Sciences, 37(2008), 1187-1192.

Faltýnová, M., Matoušková, E., Šedina, J., \& Pavelka, K. (2016). BUILDING FACADE DOCUMENTATION USING LASER SCANNING AND PHOTOGRAMMETRY AND DATA IMPLEMENTATION INTO BIM. International Archives of the Photogrammetry, Remote Sensing \& Spatial Information Sciences, 41.

Hou, S. Q. (2016). A Design and Research on Protection of Architectural Heritage Based on Digital Technology. In MATEC Web of Conferences (Vol. 63, p. 02038). EDP Sciences.

Moussa, W., Abdel-Wahab, M., \& Fritsch, D. (2012, October). Automatic fusion of digital images and laser scanner data for heritage preservation. In Euro-Mediterranean Conference (pp. 76-85). Springer, Berlin, Heidelberg.

Murtiyoso, A., Grussenmeyer, P., \& Freville, T. (2017). Close range UAV accurate recording and modeling of St-Pierre-leJeune neo-romanesque church in Strasbourg (France). The International Archives of Photogrammetry, Remote Sensing and Spatial Information Sciences, 42, 519.
Nex, F., \& Remondino, F. (2014). UAV for 3D mapping applications: a review. Applied geomatics, 6(1), 1-15.

Nouwakpo, S. K., Weltz, M. A., \& McGwire, K. (2016). Assessing the performance of structure-from-motion photogrammetry and terrestrial LiDAR for reconstructing soil surface microtopography of naturally vegetated plots. Earth Surface Processes and Landforms, 41(3), 308-322.

Quattrini, R., Pierdicca R. \& Morbidoni, C. (2017). Knowledgebased data enrichment for HBIM: Exploring high-quality models using the semantic-web. Journal of Cultural Heritage, 28.

Rock, G., Ries, J. B., \& Udelhoven, T. (2011). Sensitivity analysis of UAV-photogrammetry for creating digital elevation models (DEM). In Proceedings of Conference on Unmanned Aerial Vehicle in Geomatics.

Sun, Z., \& Cao, Y. K. (2015). Data processing workflows from low-cost digital survey to various applications: three case studies of Chinese historic architecture. The International Archives of Photogrammetry, Remote Sensing and Spatial Information Sciences, 40(5), 409.

Tang, P., Huber, D., Akinci, B., Lipman, R., \& Lytle, A. (2010). Automatic reconstruction of as-built building information models from laser-scanned point clouds: A review of related techniques. Automation in construction, 19(7), 829-843.

Themistocleous, K., loannides, M., Agapiou, A., \& Hadjimitsis, D. G. (2015). A new approach for documenting architectural cultural heritage: the case study of Asinou Church in Cyprus. In Intl Conf. on Sustainability in Arch. Cult. Hert..

Volk, R., Stengel, J., \& Schultmann, F. (2014). Building Information Modeling (BIM) for existing buildings-Literature review and future needs. Automation in construction, 38, 109127.

Xu, Z., Wu, T. H., Shen, Y., \& Wu, L. (2016). Three dimentional reconstruction of large cultural heritage objects based on UAV video and TLS data. The International Archives of Photogrammetry, Remote Sensing and Spatial Information Sciences, 41, 985 . 